RECyT

Año 23 / № 35 / 2021 / 30-34

\title{
Estudios preliminares para el procesamiento de minerales mediante calcinación solar
}

\section{Preliminary studies for the processing of minerals by solar calcination}

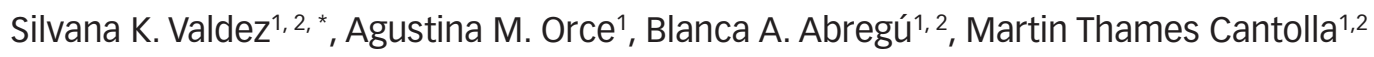 \\ 1- Facultad de Ingeniería, Universidad Nacional de Salta. Av. Bolivia 5150, 4400, Salta, Argentina. \\ 2- Instituto de Investigaciones para la Industria Química (INIQUI-CONICET), Av. Bolivia 5150, 4400, Salta, Argentina. \\ *E-mail: skvaldez@gmail.com \\ Recibido: 30/12/2019; Aprobado 23/03/2021
}

\section{Resumen}

Ciertos países actualmente cuentan con sistemas que emplean la energía solar en diversos procesos industriales. Estos sistemas tienen la ventaja de disminuir el empleo de combustibles fósiles y con ello la generación de dióxido de carbono, por lo que se trata de sistemas sustentables.

No existen antecedentes en Argentina del uso de energía solar en procesos pirometalúrgicos. En este trabajo se analiza la factibilidad de realizar la calcinación de boratos empleando energía solar. La calcinación de un borato consiste en su descomposición térmica, eliminándose su agua de hidratación e incrementando su contenido de $\mathrm{B}_{2} \mathrm{O}_{3}$. En el caso particular de la colemanita (borato de calcio), la temperatura de descomposición se encuentra entre $350-400^{\circ} \mathrm{C}$. Se calcinó una muestra de colemanita del $70 \%$ de pureza en una pantalla solar de $172 \mathrm{~cm}$ de diámetro y profundidad de foco de $40 \mathrm{~cm}$ con un ángulo de inclinación de $30^{\circ}$. Se determinó la irradiación $\left(\mathrm{kcal} / \mathrm{m}^{2}\right.$ h) interceptada por el equipo. Dependiendo de las condiciones climáticas se obtuvo una conversión entre 45-72\%. Además se midió el grado de avance a distintos periodos de tiempo durante una hora. Los resultados demuestran que es posible utilizar la energía solar como fuente de energía alternativa para calcinar colemanita.

Palabras clave: Colemanita; Calcinación; Concentración; Energía Solar.

\section{Abstract}

Certain countries have systems using solar energy on industrial process. The advantage of these systems is to reduce the fossil fuel consumption and carbon dioxide generation, being a sustainable system. There is no background information about the use of non-conventional energy sources applied to pyrometallurgical processes in Argentina. In this paper the feasibility of calcining borates by solar energy was analyzed. The calcination of borates allows to increase their $\mathrm{B}_{2} \mathrm{O}_{3}$ content due to the thermal decomposition with the loss of the hydration water. In particular, the calcination of colemanite (calcium borate) occurs around $350-400^{\circ} \mathrm{C}$. A colemanite sample of $70 \%$ purity was calcined using a dish concentrator of $172 \mathrm{~cm}$ diameter and a focus depth of $40 \mathrm{~cm}$, the rim angle was $30^{\circ}$. The radiation intercepted by the equipment was determined in $\mathrm{kcal} / \mathrm{m}^{2} \mathrm{~h}$. Conversion was between $45-72 \%$ depending on the environmental conditions. The reaction advancement was measured at different periods of time within an hour. Results show that it is possible to use solar radiation as energy source to calcine and purify colemanite.

Keywords: Colemanite; Calcining; Concentration; Solar Energy.

\section{Introducción}

Numerosos procesos industriales demandan energía térmica en el rango de $80-240^{\circ} \mathrm{C}$ [1]. En Sharma et al. (2017) [2], se recopiló información sobre el desarrollo de sistemas de calefacción de procesos industriales mediante energía solar. Países como India, China, Estados Unidos, Alemania, emplean sistemas de energía térmica en industrias agropecuarios, alimenticias, textil, entre otras [3]. Estos sistemas no son tenidos en cuenta en la industria minera ya que se requieren elevadas temperaturas de procesamiento [3].

Argentina es uno de los pocos países que dispone de variados yacimientos de boratos: los denominados "duros" (tincal, colemanita, hidroboracita), así como boratos contenidos en la costra de los salares (ulexita) o disueltos en la salmuera impregnante. Es la tercera exportadora mundial de productos del boro.

El procesamiento de los boratos depende de las propiedades y de la pureza deseada en el producto final. En sen- 
tido creciente de valor los principales productos del boro son: mineral sin procesar, concentrados, calcinados, fritas, refinados y BAVA (Boratos de Alto Valor Agregado). Dado el elevado contenido de agua de hidratación de los boratos, la calcinación se muestra como una interesante alternativa para elevar la ley (contenido en $\mathrm{B}_{2} \mathrm{O}_{3}$ ) de minerales o de sus concentrados.

La calcinación consiste en una descomposición térmica del borato, que elimina su agua de hidratación incrementando apreciablemente el contenido de $\mathrm{B}_{2} \mathrm{O}_{3}$. Es un proceso que se realiza a relativamente baja temperatura [4]. Los tratamientos pirometalúrgicos de calcinación y de anhidración poseen además una importante ventaja adicional: disminuye el costo de transporte, ítem de suma importancia para los boratos, cuyos yacimientos están generalmente ubicados lejos de los puertos y muy lejos de los mercados, afectando fuertemente la competitividad del sector.

En el caso de la colemanita (borato de calcio), su calcinación ocurre de manera violenta: decrepita, disminuyendo notoriamente su tamaño de partícula hasta tamaño de polvo; simultáneamente también ocurre una disminución de su peso específico, desde 2,4 a 1,9 [5].

La descomposición térmica ocurre alrededor de los 300$450^{\circ} \mathrm{C}[5,6,7]$. Como la ganga permanece inalterada tanto en tamaño como en peso específico, ocurre un incremento en la diferencia de esas propiedades que mejora las condiciones de concentración respecto de la carga sin calcinar. Celik y Cakal [5] y Şenol-Arslan et al. [8] estudiaron cómo varían las propiedades físicas con el tratamiento térmico de muestras de colemanita provenientes de diversos yacimientos de Turquía. Can et al. [9] realizaron la calcinación de colemanita empleando un horno de microondas doméstico. Encontraron que el método es ventajoso en cuanto al tiempo de procesamiento $\left(10\right.$ minutos para alcanzar $\left.450^{\circ} \mathrm{C}\right) \mathrm{y}$ por una reducción de $100^{\circ} \mathrm{C}$ en la temperatura de transición de la colemanita a colemanita amorfa.

En Argentina, los yacimientos de boratos se localizan en la región de la Puna, a más de 3000 metros sobre el nivel del mar. Esta región se caracteriza por tener condiciones climáticas extremas, altos gradientes térmicos entre el día y la noche, sol diáfano y escasas precipitaciones durante el año. La probabilidad de días soleados en el invierno es de $85 \%$.

La irradiación (o insolación) es la cantidad de radiación global recibida en un punto geográfico durante un tiempo determinado y sobre una superficie conocida $\left(\mathrm{kWh} / \mathrm{m}^{2}\right)$ y la irradiancia es la magnitud utilizada para describir la potencia incidente sobre unidad de superficie de todo tipo de radiación electromagnética $\left(\mathrm{W} / \mathrm{m}^{2}\right)$. En la región la irradiación varía desde 5,5 a $7,5 \mathrm{kWh} / \mathrm{m}^{2}$ en enero, mientras que en junio lo hace entre 4,5 y $5,5 \mathrm{kWh} / \mathrm{m}^{2}$; en los mismos meses la heliofanía varía, en promedio, entre 8-9 horas [10]. En Salta, en días soleados la irradiancia es de 900 $\mathrm{W} / \mathrm{m}^{2}[11]$.

No existen antecedentes en nuestro país del uso de ener- gía no convencional en procesos pirometalúrgicos, sin embargo las condiciones climáticas en la Puna hacen suponer que es factible el uso de la energía solar para llevar a cabo procesos térmicos de relativamente baja temperatura, como en el beneficio de los boratos. En este trabajo se analiza la factibilidad de realizar la calcinación de colemanita (borato de calcio) empleando un concentrador parabólico solar.

\section{Materiales y Métodos}

Para la calcinación se empleó un concentrador solar parabólico de $172 \mathrm{~cm}$ de diámetro y profundidad de foco (distancia focal) de $40 \mathrm{~cm}$, con un ángulo de inclinación de $30^{\circ}$.

Las muestras de colemanita empleadas tenían 35,58\% $\mathrm{B}_{2} \mathrm{O}_{3}, 19,09 \% \mathrm{CaO}$ y el $45,33 \%$ restante corresponde a insolubles tales como sílice. La colemanita pura contiene $50,81 \% \mathrm{~B}_{2} \mathrm{O}_{3}$ por lo que las muestras empleadas tenían una pureza del 70\%. Las muestras de tamaño entre 8-10 $\mathrm{M}(2,38-2 \mathrm{~mm})$ y se calcinaron entre 60 y 120 minutos. Durante el proceso de deshidratación las partículas decrepitan reduciéndose su tamaño, en consecuencia luego de los ensayos las partículas tenían un tamaño $<20 \mathrm{M}(<0,84$ $\mathrm{mm}$ ). Las muestras se pesaron antes y después de cada ensayo para determinar la pérdida de peso. Se registró la temperatura a cada instante de tiempo mediante dos termocuplas ubicadas a dos alturas diferentes separadas $7 \mathrm{~mm}$. Se realizaron además ensayos para determinar el avance de la reacción en función del tiempo. Para ello se emplearon muestras cuya masa se informa en la Tabla 2 y se repitieron los mismos a distintos tiempos entre 3 minutos y una hora. Se analizó el contenido de $\mathrm{B}_{2} \mathrm{O}_{3}$ antes y después de la calcinación mediante análisis químico por titulación con $\mathrm{Na}(\mathrm{OH})$ [4].

Para determinar la energía radiante incidente en la muestra $\left(\mathrm{q}_{\mathrm{ri}}\right)$ se realizaron ensayos de vaporización de agua. Para ello se colocó en el foco del concentrador un recipiente con agua a vaporizar. Los ensayos se realizaron empleando recipientes de vidrio, hierro fundido y acero ya que $\mathrm{q}_{\mathrm{ri}}$ depende de las propiedades ópticas del material que recibe la radiación.

La temperatura se registró a distintos tiempos. Los ensayos se realizaron a las 9 de la mañana y tuvieron una duración de una hora. En todos los ensayos el concentrador se ubicó de cara al Este, se considera que en una hora no hay variación en el ángulo de incidencia de la radiación sobre el recipiente portamuestra ya que la pantalla se ajustaba en cada instante de tiempo manualmente para mantener el foco centrado.

El calor útil se define, como la diferencia entre el calor interceptado por el portamuestra y las pérdidas por radiación y convección, de acuerdo a la ecuación 1 . 
$Q_{u}=Q_{r i}-A_{r p}\left[\sigma\left(T^{4}-T_{\infty}^{4}\right)+h\left(T-T_{\infty}\right)\right]$

donde:

$Q_{u}:$ Calor útil, kcal/h

$Q_{r i}$ : Calor debido a la radiación reflejada por el concentrador e interceptada por el portamuestra, $\mathrm{kcal} / \mathrm{h}$

$A_{r p}$ : Área de incidencia en el recipiente portamuestra, $\mathrm{m}^{2}$

$\sigma$ : Constante de Boltzman, $3,304 \times 10^{-27} \mathrm{kcal} / \mathrm{K}$

$\mathrm{T}$ : temperatura, $\mathrm{K}$

$T_{\infty}$ : Temperatura ambiente, $\mathrm{K}$

$\mathrm{h}$ : coeficiente pelicular de transferencia de calor, $\mathrm{kcal} / \mathrm{h}$ $\mathrm{m}^{2} \mathrm{~K}$

A su vez, $Q_{r i}$ depende de la eficiencia óptica del material $\left(\epsilon_{0}, \%\right)$, del área de apertura del concentrador parabólico $\left(A_{a}, \mathrm{~m}^{2}\right)$ y de la irradiancia $\left(I_{b}, \mathrm{~W} / \mathrm{m}^{2}\right)$ (ecuación 2$)$.

$$
Q_{r i}=\frac{\epsilon_{0} A_{a} I_{b}}{1,163}
$$

La densidad de calor incidente $\left(\mathrm{q}_{\mathrm{ri}}\right)$ se define en la ecuación 3:

$$
q_{r i}=\frac{Q_{r i}}{A_{r p}}
$$

Conocido el calor útil y las temperaturas se puede calcular el $Q_{r i}$ (ecuación 1) y con él la eficiencia óptica $\epsilon_{0}$ (ecuación 2).

Por último, la eficiencia térmica instantánea se define de acuerdo a la ecuación 4 como:

$$
=\frac{Q_{u} 1,163}{I_{b} A_{a}}
$$

Para determinar el calor interceptado $\left(\mathrm{Q}_{\mathrm{ri}}\right)$ se vaporizó una cierta cantidad de agua contenida en distintos recipientes: de vidrio (diámetro $18 \mathrm{~cm}$ ), de acero inoxidable (diámetro $10 \mathrm{~cm}$ ) y de hierro fundido (diámetro $24 \mathrm{~cm}$ ). Como se conoce la cantidad de agua evaporada y el calor latente de vaporización a presión atmosférica, el calor incidente se calculó con la ecuación 5 .

$$
Q_{u}=\frac{m_{H_{2} O} \Delta H_{v a p}+m_{H_{2} O} C_{e} \Delta T}{t}
$$

Siendo t el tiempo de exposición expresado en horas.

\section{Resultados y Discusión}

En la Tabla 1 se presentan los resultados de flujo de calor y densidad de flujo de calor interceptado así como también los parámetros del concentrador solar. La relación $\mathrm{q}_{\mathrm{ri}} / \mathrm{I}_{\mathrm{b}}$ es una medida de cuánto concentra el equipo la radiación solar.
Tabla 1: Parámetros del concentrador solar.

\begin{tabular}{|c|c|c|c|c|c|}
\hline $\begin{array}{c}\text { Evaporación solar de agua. } \\
\text { Equipos empleados }\end{array}$ & $\mathbf{Q}_{\mathrm{r},}, \mathbf{k c a l} / \mathbf{h}$ & $\begin{array}{r}\mathbf{q}_{\mathrm{ri}} \\
\mathbf{k c a l} /\left(\mathbf{h ~ m}^{2}\right)\end{array}$ & $\varepsilon_{0}$ & $\eta$ & $\mathbf{q}_{\mathrm{r}} / \mathbf{I}_{\mathbf{b}}$ \\
\hline $\begin{array}{c}\text { Cristalizador vidrio } \\
(\mathrm{f}=18,5 \mathrm{~cm}) \mathrm{h}=8,5 \mathrm{~cm}\end{array}$ & 585,7 & 7681,7 & 32,62 & 27,79 & 9,93 \\
\hline $\begin{array}{c}\text { Hierro Fundido negro } \\
(\mathrm{f}=24 \mathrm{~cm}) \mathrm{h}=11 \mathrm{~cm}\end{array}$ & 971,5 & 7582,8 & 54,11 & 46,32 & 9,80 \\
\hline $\begin{array}{c}\text { Acero inoxidable } \\
(\mathrm{f}=10 \mathrm{~cm}) \mathrm{h}=1,3 \mathrm{~cm} \text { c/agua }\end{array}$ & 191,0 & 12167,1 & 10,64 & 9,93 & 15,72 \\
\hline $\begin{array}{c}\text { Acero inoxidable } \\
(\mathrm{f}=10 \mathrm{~cm}) \mathrm{h}=1,3 \mathrm{~cm} \text { c/agua }\end{array}$ & 217,3 & 13839,9 & 12,10 & 11,47 & 17,88 \\
\hline $\begin{array}{c}\text { Acero inoxidable } \\
(\mathrm{f}=10 \mathrm{~cm}) \mathrm{h}=1,3 \mathrm{~cm} \text { c/agua }\end{array}$ & 205,1 & 13066,6 & 11,43 & 10,56 & 16,88 \\
\hline
\end{tabular}

El calor interceptado depende de las propiedades del recipiente, en la Tabla 1 se observa que tanto el cristalizador de vidrio como el recipiente de hierro fundido tienen densidad de calor interceptado cercano a $7600 \mathrm{kcal} / \mathrm{h} \mathrm{m}^{2}$. Los ensayos empleando el mismo recipiente de acero inoxidable se llevaron a cabo en distintos días por lo que los valores de la densidad de calor incidente $\left(\mathrm{q}_{\mathrm{ri}}\right)$ varían entre ensayos. Comparándolo con los demás recipientes, el de acero inoxidable tiene una densidad mayor, alcanzando valores promedio de $13.000 \mathrm{kcal} / \mathrm{h} \mathrm{m}^{2}$. La diferencia se debe a las pérdidas por radiación y convección en cada caso, según se observa en la ecuación 1. Por este motivo los ensayos de calcinación de colemanita se llevaron a cabo empleando los recipientes de acero inoxidable.

En la Figuras 1a y 1 b se observa la variación de temperatura superior e inferior, medidas con dos termocuplas separadas $7 \mathrm{~mm}$. La termocupla superior se ubicó debajo de una capa de $3 \mathrm{~mm}$ de mineral. Las figuras corresponden a los Ensayos 1 y 3 respectivamente (ver Tabla 2). En ambos ensayos se alcanza la temperatura necesaria para producir la decrepitación de la colemanita. La diferencia de temperaturas entre las termocuplas (DT), considerando la distancia entre ellas $(7 \mathrm{~mm})$, indicaría una baja transmisión de calor en el sentido vertical, por lo que se podría asumir el fenómeno como un proceso superficial que no se propaga hacia el interior de la muestra. El descenso brusco de las temperaturas en la Figura 1a se debió a la presencia de nubosidad.

Se observa que en ensayos desarrollados en condiciones operativas similares, se alcanzan temperaturas muy diferentes. Esto es debido a que las condiciones ambientales no se mantienen constantes, y a que el movimiento de la pantalla para mantener el foco centrado se realizó manualmente. 


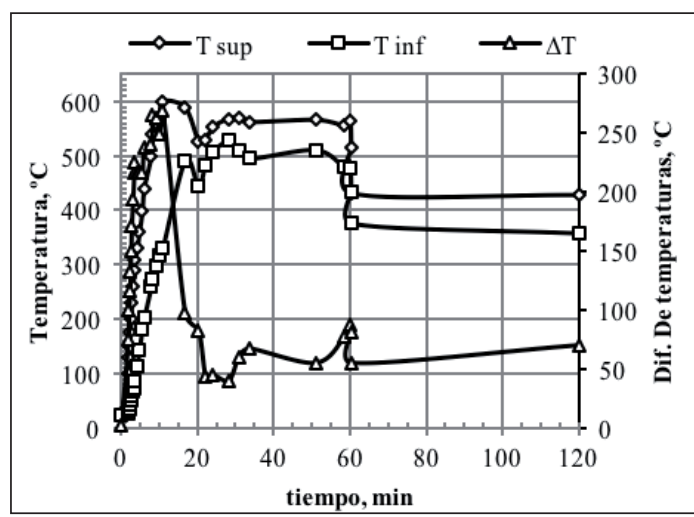

Figura 1a: Evolución de la temperatura durante la calcinación (Ensayo 1).

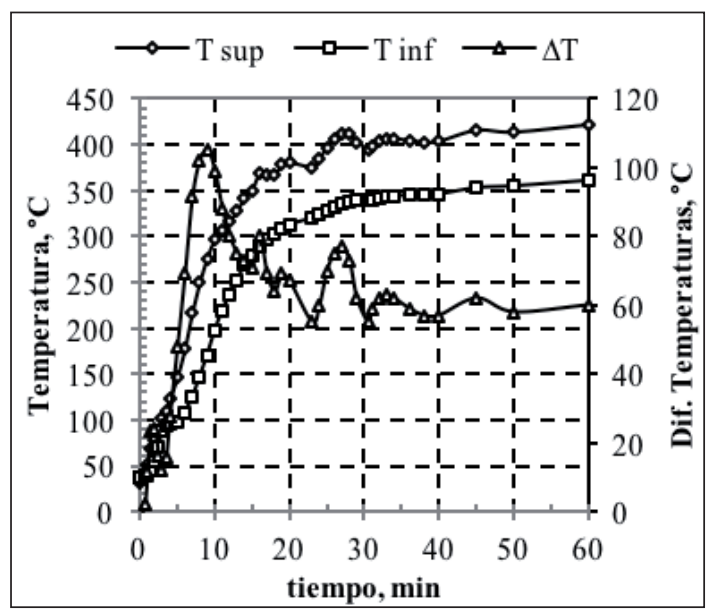

Figura 1b: Evolución de la temperatura durante la calcinación (Ensayo 3).

En Tabla 2 se muestran los resultados de tres ensayos de calcinación realizados en tiempos suficientemente largos tal que se supone que se alcanza el máximo avance posible de la reacción para las condiciones operativas empleadas.

Tabla 2: Resultados de ensayos de calcinación solar.

\begin{tabular}{|c|c|c|c|}
\hline Ensayo & $\begin{array}{c}\mathbf{1} \\
\mathbf{t}: \mathbf{1 2 0} \mathbf{m i n}\end{array}$ & $\begin{array}{c}\mathbf{2} \\
\text { t:100 } \mathbf{~ m i n}\end{array}$ & $\begin{array}{c}\mathbf{3} \\
\mathbf{t}: 60 \text { min }\end{array}$ \\
\hline $\begin{array}{c}\text { Tamaño de colemanita } \\
\left(35,58 \% \mathrm{~B}_{2} \mathrm{O}_{3}\right) \text { alimentada }\end{array}$ & $8-10 \mathrm{M}$ & $8-10 \mathrm{M}$ & $8-10 \mathrm{M}$ \\
\hline Masa inicial; $\mathrm{g}$ & 92,5 & 104,2 & 104 \\
\hline Masa final; $\mathrm{g}$ & 82,24 & 95,7 & 93,42 \\
\hline Pérdida de peso $(\Delta \mathrm{W}) ; \%$ & 11,09 & 8,16 & 10,17 \\
\hline Grado avance $(\eta) ; \%$ & 67,44 & 49,60 & 61,82 \\
\hline Contendido de $\mathrm{B}_{2} \mathrm{O}_{3}$ en $>20 \mathrm{M} ; \%$ & 37,01 & 37,88 & 36,48 \\
\hline Contenido de $\mathrm{B}_{2} \mathrm{O}_{3}$ en $<20 \mathrm{M} ; \%$ & 46,56 & 44,31 & 46,19 \\
\hline Recuperación en $<20 \mathrm{M} ; \%$ & 34,28 & 21,83 & 33,50 \\
\hline
\end{tabular}

La ley de $\mathrm{B}_{2} \mathrm{O}_{3}$ creció de $35,58 \%$ en la alimentación a un 44,3-46,6\% en la fracción $<20 \mathrm{M}$ con un grado de avance entre $50-67 \%$. La fracción $>20 \mathrm{M}$ aumenta levemente el contenido de $\mathrm{B}_{2} \mathrm{O}_{3}$ pero es una fracción que además de ganga, también posee colemanita sin reaccionar. En el Ensayo 1 las temperaturas alcanzadas durante el tiempo de reacción máximo ensayado $(120$ minutos $) \approx 600^{\circ} \mathrm{C}$ son del orden de las temperaturas alcanzadas en los hornos tradicionales. Esta temperatura, aún cuando se mantuvo durante aproximadamente 60 minutos no fue suficiente para alcanzar la conversión deseada. Por otro lado, la colemanita comienza a fundir a $1.015^{\circ} \mathrm{C}$ por lo que no ocu- rrieron reacciones secundarias durante el ensayo. A pesar de haber permanecido tiempo suficiente no se alcanzan valores de conversión muy elevados. Esto demuestra que la reacción ocurre en la superficie expuesta a la radiación y que el mineral sin reaccionar de las capas inferiores ofrece una alta resistencia a la transferencia de calor, lo mismo se aprecia con la diferencia de temperaturas entre las termocuplas ubicadas a una distancia de $7 \mathrm{~mm}$ entre sí.

Se realizaron también seis calcinaciones para analizar la influencia del tiempo a 3, 7, 10, 20, 30 y 60 minutos. En la Tabla 3 se muestran las masas iniciales y finales mientras que en la Figura 2 muestra la influencia del tiempo sobre la pérdida de peso, el grado de avance y la recuperación de $\mathrm{B}_{2} \mathrm{O}_{3}$ en la fracción fina $(<20 \mathrm{M})$.

Los resultados obtenidos son comparables con aquellos que se muestran en la Tabla 1. La máxima velocidad de cambio de las tres variables estudiadas ocurre durante los primeros 10 minutos de calcinación. Esto también demuestra la dificultad en la transferencia de calor hacia el interior de la muestra.

Tabla 3: Masa inicial y masa final medida a diferentes tiempos.

\begin{tabular}{|c|c|c|c|c|c|c|}
\hline Ensayo & $\begin{array}{c}\mathbf{1} \\
\text { t:3 } \mathbf{~ m i n}\end{array}$ & $\begin{array}{c}\mathbf{2} \\
\text { t:7 } \mathbf{~ m i n}\end{array}$ & $\begin{array}{c}\mathbf{3} \\
\text { t:12 } \mathbf{m i n}\end{array}$ & $\begin{array}{c}\mathbf{1} \\
\text { t:20 } \mathbf{~ m i n}\end{array}$ & $\begin{array}{c}\mathbf{2} \\
\text { t:30 } \mathbf{m i n}\end{array}$ & $\begin{array}{c}\mathbf{3} \\
\text { t:60 } \mathbf{~ m i n}\end{array}$ \\
\hline $\begin{array}{c}\text { Tamaño de } \\
\text { colemanita } \\
\left(35,58 \% \mathrm{~B}_{2} \mathrm{O}_{3}\right) \\
\text { alimentada }\end{array}$ & $8-10 \mathrm{M}$ & $8-10 \mathrm{M}$ & $8-10 \mathrm{M}$ & $8-10 \mathrm{M}$ & $8-10 \mathrm{M}$ & $8-10 \mathrm{M}$ \\
\hline Masa inicial; $\mathrm{g}$ & 97,55 & 98,07 & 97,94 & 96,92 & 96,30 & 95,79 \\
\hline Masa final; g & 95,95 & 95,38 & 93,01 & 91,30 & 89,88 & 88,61 \\
\hline
\end{tabular}

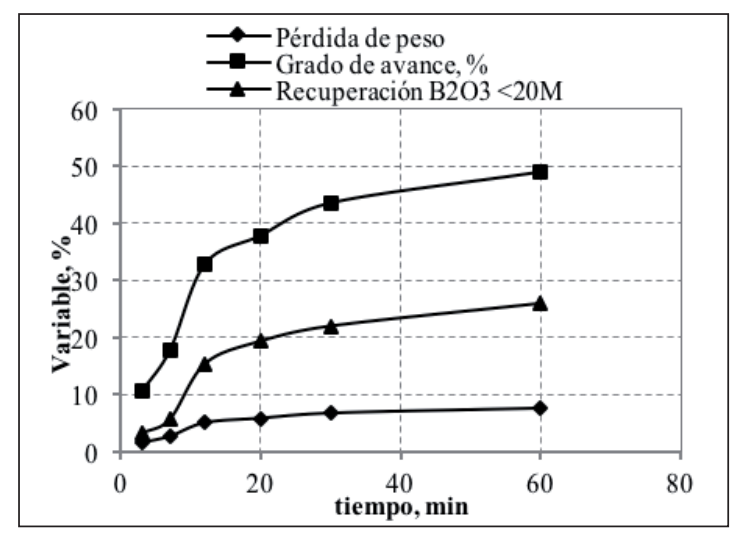

Figura 2: Evolución de la calcinación en el tiempo.

\section{Conclusiones}

Es factible calcinar colemanita empleando un concentrador solar. Dependiendo de las condiciones climáticas se obtuvo una conversión entre 45-72\%. El calentamiento ocurre en la superficie y la temperatura no se transmite fácilmente hacia el seno del lecho mineral. La irradiancia media anual de la Puna es más que suficiente para alcanzar las temperaturas de decrepitación de la colemanita; ésto, sumado a que la mayoría de las empresas borateras realizan la calcinación en plantas cercanas a los yacimientos hace 
que el proceso de calcinación pueda llevarse a cabo durante todo el año. El proceso será discontinuo y se recomienda trabajar durante las 9 horas de heliofanía de la región.

Para mejorar la transferencia, y en consecuencia la conversión, se debe diseñar un sistema un sistema dinámico que permita que la totalidad de las partículas se encuentren expuestas a la radiación. Los resultados alientan a continuar con la investigación orientada al diseño de un reactor experimental a escala banco-piloto que permita la obtención de índices metalúrgicos de interés industrial.

\section{Referencias Bibliográficas}

1. Kulkarni, G. et al. (2008). Design of solar thermal systems utilizing pressurized hot water storage for industrial applications. Solar Energy Vol. 82. pp 686-699.

2. Sharma, A. et al. (2017). Solar industrial process heating: A review. Renewable and Sustainable Energy Reviews Vol. 78, pp124-137.

3. Farjana, s. et al. (2018) Solar industrial process heating systems in operation - Current SHIP plants and future prospects in Australia. Renewable and Sustainable Energy Reviews 91, pp 409-419.

4. Uysal, T. et al. (2016).Effects of mechanical activation of colemanite (Ca2B6O11.5H2O) on its thermal transformations. International Journal of Mineral Processing 151, pp 51-58
5. Flores, H. (2004) Beneficio de los Boratos del NOA. Editorial Crisol. 2004

6. Celik, A. and Cakal, G. (2016). Characterization of espey colemanite and variation of its physical properties with temperature. Physicochemical Problems of Mineral Processing 52 (1); pp 66-76.

7. Guzel, G. et al. (2016). The use of colemanite and ulexite as novel fillers in epoxy composites: Influences on thermal and physico-mechanical properties. Influences on thermal and physico-mechanical properties. Composites Part B 100, pp1-9

8. Şenol-Arslan, D. et al. (2016). Investigation of Thermal Behaviour of Hisarcik and Espey Colemanites. Bildiriler Kitabı, TMMOB Metalurji ve Malzeme Mühendisleri Odas1, UCTEA Chamber of Metallurgical \& Materials Engineers Proceedings Book, Uluslararası Metalurji ve Malzeme Kongresi | IMMC; pp 431-434.

9. Fatih Can, M. et al. (2016) Microwave Assisted Calcination of Colemanite Powders. International Journal of Metallurgical \& Materials Engineering.

10. Grossi Gallegos, H. y R. Righini. (2007) Atlas de la Energía Solar de la República Argentina. Disponible en: www. gersolar.unlu.edu.ar.

11. Piacentini, R. (2003) Introducción a la energía del sol y la eficiencia energética. Concursol centro Científico Tecnológico. Rosario, CONICET. 